\title{
A MODEL BASED UPON THE CROSS-CORRELATION FUNCTION BETWEEN THE POTENTIAL AND WAVE FUNCTIONS FOR DISORDERED SOLIDS
}

\author{
M. A. GRADO-CAFFARO* and M. GRADO-CAFFARO \\ Scientific Consultants, C/Julio Palacios, 11, 9-B, 28029-Madrid, Spain
}

(Received 12 February 1997; In final form 15 April 1998)

\begin{abstract}
A formulation for the cross-correlation function between the potential and Schrödinger wave functions with respect to the spacing between consecutive atoms in an infinite onedimensional disordered solid is proposed. In this theoretical model, the argument of the above cross-correlation function is the spacing in question which is regarded as a random variable.
\end{abstract}

Keywords: Cross-correlation function; Schrödinger wave functions; disordered solids

\section{INTRODUCTION}

It is well-known that a solid in crystalline state presents a constant spacing between adjacent atoms; this spacing is the period of the corresponding crystalline lattice. On the other hand, it is also wellknown that a given electron in the solid is submitted to a periodic field whose potential energy function is a periodic function whose period coincides with the above spacing. If we consider this spacing as a random variable, we are considering implicitly the solid as a disordered system. In addition, we can claim that a measure of this randomness is a certain correlation function. For clear reasons which will be given later, we shall choose as this function the cross-

\footnotetext{
*Corresponding author.
} 
correlation function between the potential function and a generic wavefunction obeying the non-relativistic time-independent Schrödinger equation. Our model deals with one-dimensional disordered solids.

\section{THEORY}

First of all, let us consider the non-relativistic time-independent Schrödinger equation in one dimension, namely:

$$
\frac{d^{2}}{d x^{2}}(\psi(x))+2 m \hbar^{-2}[E-V(x)] \psi(x)=0
$$

From now on we shall assume an infinite and one-dimensional disordered solid so that from Eq. (1) and considering that $V(x)=V(x+s)(V$ denotes potential energy and $s$ is lattice period $)$, we get:

$$
\frac{d^{2}}{d x^{2}}(\psi(x+s))+2 m \hbar^{-2}[E-V(x)] \psi(x+s)=0
$$

since $\psi(x+s)$ obeys Eq. (1) (we assume real $\psi(x)$ ).

From expression (2), it follows:

$$
\begin{aligned}
\xi(s) \equiv & \int_{0}^{\infty} V(x) \psi(x+s) d x=\frac{\hbar^{2}}{2 m}\left[\left.\frac{d}{d x}(\psi(x))\right|_{x \rightarrow \infty}-\frac{d}{d s}(\psi(s))\right] \\
& +E \int_{s}^{\infty} \psi(x) d x
\end{aligned}
$$

where $\xi(s)$ is the cross-correlation function between $V$ and $\psi$.

Observing relationship (3), we see that the existence of $\xi(s)$ is assured if the Schrödinger eigenfunctions are integrable. Even this condition does not arise from the well-known square-integrability of $\psi(x)$, in practice, the major part of the typical cases in solid state physics (in particular, physics of semiconductors) exhibit the integrability of $\psi(x)$. On the other hand, at this point we want to emphasize that we have assumed $\psi(x)$ as real functions which constitutes a typical situation in practice. 
Now, from Eq. (3) it follows:

$$
\frac{d}{d s}(\xi(s))=-E \psi(s)-\frac{\hbar^{2}}{2 m} \frac{d^{2}}{d s^{2}}(\psi(s))
$$

In addition, by taking into account expression (3), the expected value of $\xi$ (or average value) is: $\bar{\xi}=\int_{0}^{\infty} \xi(s) \chi(s) d s$ so that

$$
\bar{\xi}=\frac{\hbar^{2}}{2 m}\left[\left.\frac{d}{d x}(\psi(x))\right|_{x \rightarrow \infty}-\overline{\left.\frac{d}{d s}(\psi(s))\right]}+E \int_{s_{1}}^{s_{2}} \chi(s) d s \int_{s}^{\infty} \psi(x) d x\right.
$$

where $\chi(s)$ is the probability density function of $s\left(s_{1} \leq s \leq s_{2}\right)$.

Finally, we can establish the following reasonable statement: the expected value of $s$ should coincide with the period of the crystalline counterpart (this period is, of course, fixed); let us denote this period by $d$. Then, we can write:

$$
\int_{s_{1}}^{s_{2}} s \chi(s) d s=d
$$

\section{CONCLUDING REMARKS}

The above formulae constitute a theoretical background to treat disordered solids with a special methodology based upon concepts related to signal theory. In this context, the cross-correlation function plays an important role; details about the physical meaning of this function can be found. for example, in Refs. [1-4]. In particular, expression (4) is very interesting. Finally, we can claim that randomness, which is a basic concept in the field of disordered solids, may be conceived in unusual ways in the light of the above exposition.

\section{References}

[1] Hecht, E. and Zajac, A., Optics (Addison-Wesley, 1974).

[2] Franks, L. E., Signal theory (Prentice-Hall, 1975).

[3] Davenport, W. B. Jr., and Root, W. L., An introduction to the theory of random signals and noise (McGraw-Hill, 1958).

[4] Grado-Caffaro, M. A. and Grado-Caffaro, M. (unpublished results). 

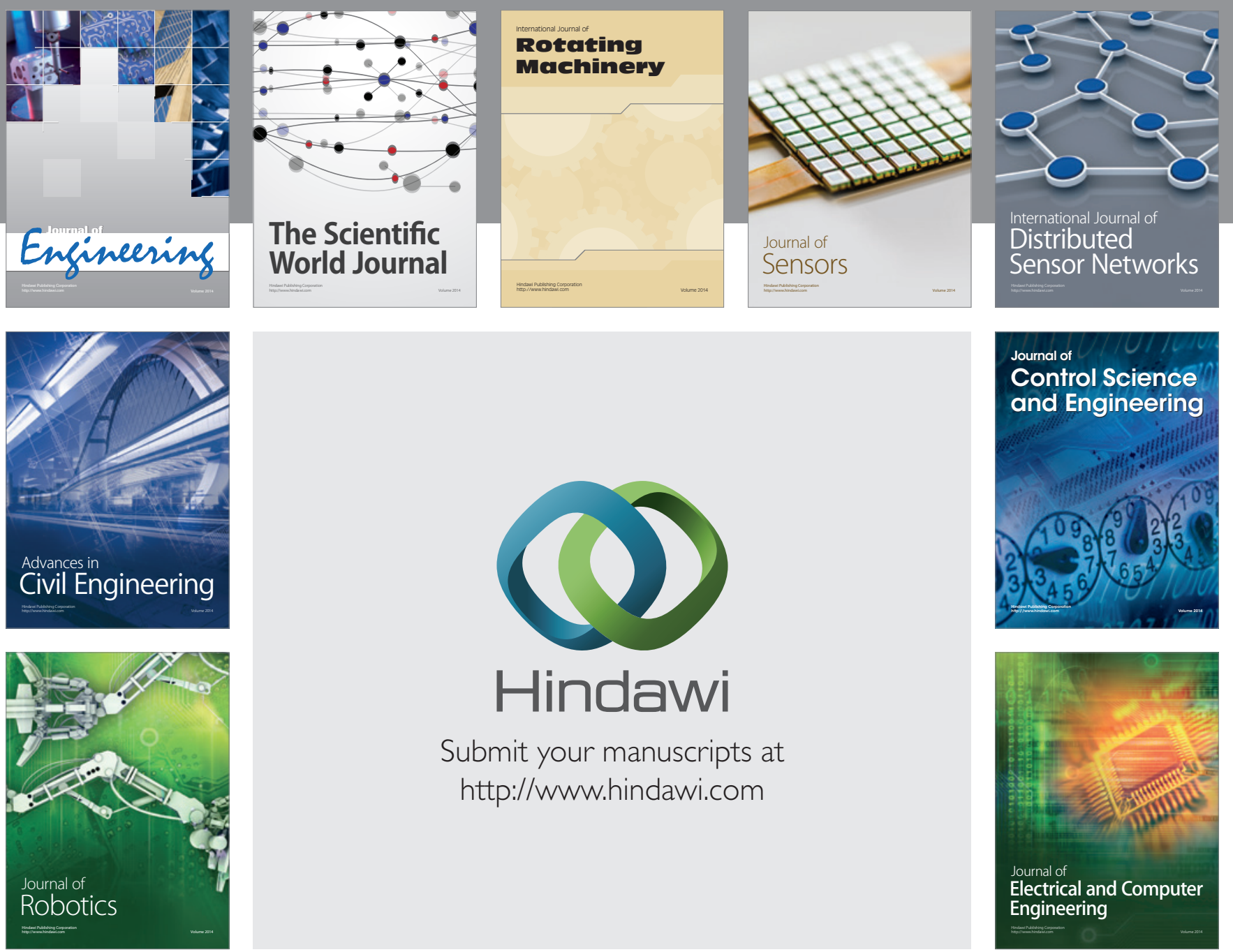

Submit your manuscripts at

http://www.hindawi.com
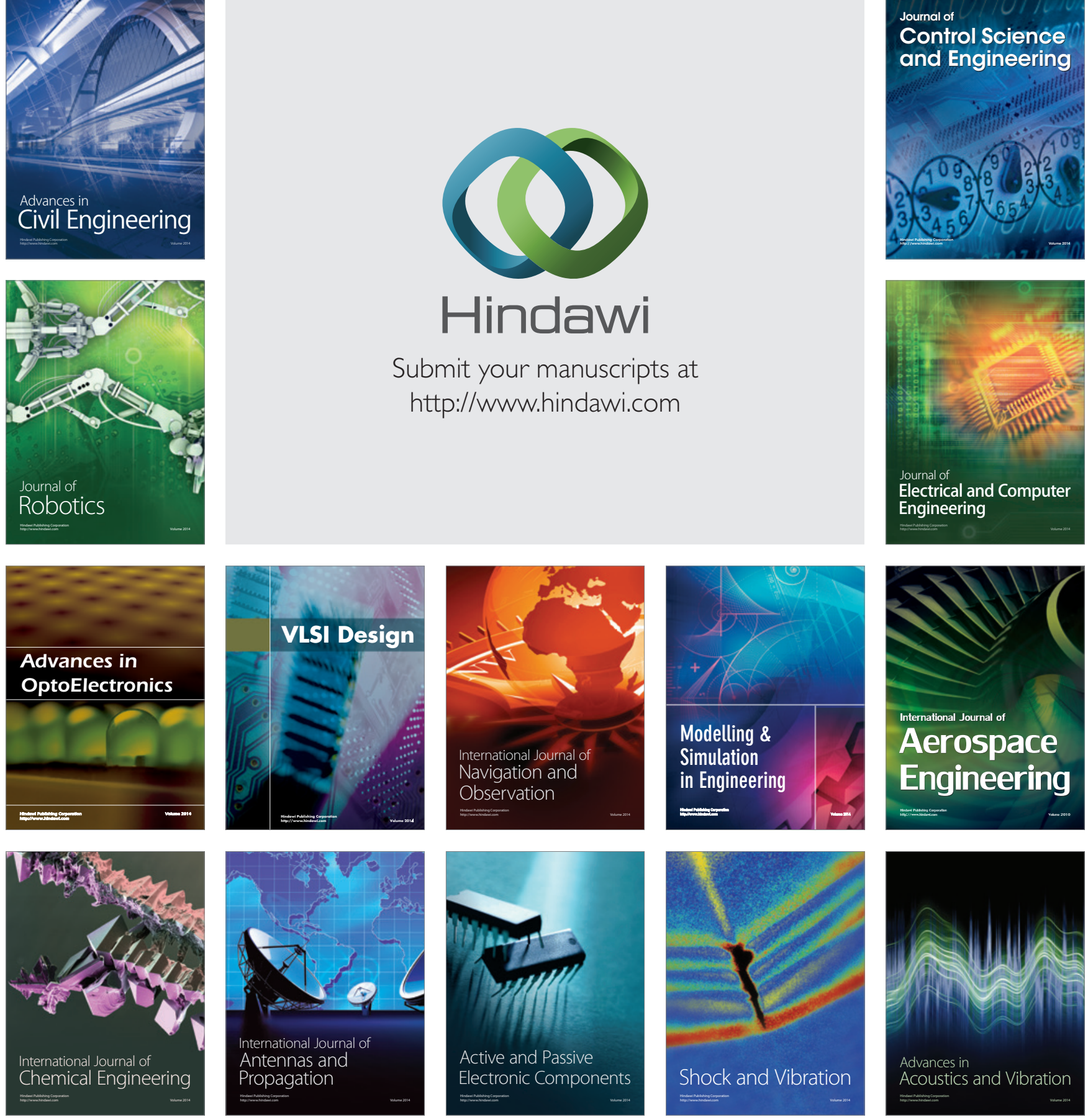